\title{
Programas multiprofissionais de tratamento da obesidade com metas de perda de peso definidas: uma revisão sistemática da literatura
}

\author{
Multiprofessional programs for treating obesity with defined weight loss targets: a systematic
} literature review

\author{
Programas multiprofisionales para el tratamiento de la obesidad con objetivos definidos de pérdida \\ de peso: revisión sistemática de la literatura
}

Recebido: 07/01/2021 | Revisado: 09/01/2021 | Aceito: 09/01/2021 | Publicado: 10/01/2021

Igor Alisson Spagnol Pereira

ORCID: https://orcid.org/0000-0001-7340-3909 Universidade Estadual de Maringá, Brasil

E-mail: igorspagnol2@hotmail.com

Fernando Malentaqui Martins

ORCID: https://orcid.org/0000-0003-1623-2183 Universidade Estadual de Maringá, Brasil

E-mail: nandoesporte1@gmail.com

Greice Westphal

ORCID: https://orcid.org/0000-0001-9107-0108 Universidade Estadual de Maringá, Brasil

E-mail: greicewes@gmail.com

Mario Moreira Castilho

ORCID: https://orcid.org/0000-0002-4855-8236 Universidade Estadual de Maringá, Brasil E-mail: mmcastilho_1905@hotmail.com

Nelson Nardo Junior

ORCID: https://orcid.org/0000-0002-6862-7868 Universidade Estadual de Maringá, Brasil

E-mail: nnjunior@uem.br

\begin{abstract}
Resumo
Introdução: O aumento do sobrepeso, obesidade e obesidade severa no mundo representam um grande desafio de saúde pública tanto em termos de prevenção quanto de tratamento da obesidade e das doenças crônicas associadas. Objetivo: Esta revisão analisa os trabalhos multiprofissionais cujas metas incluam a perda de peso de, pelo menos, $5 \%$ usadas em programas que visam a mudança de comportamento em pessoas adultas com IMC acima de $30 \mathrm{~kg} / \mathrm{m}^{2} \mathrm{bem}$ como seus desfechos clínicos. Métodos: Foi realizada uma revisão sistemática nas bases de dados Pubmed e Embase com os descritores "multi-professional", "treatment", "primary care" e "obesity", nas duas bases foram utilizados os filtros "ensaio clínico", "estudos randomizados" e "adultos maiores de 18 anos", os trabalhos selecionados deveriam ter avaliado programas de intervenção presencial, com meta de perda de peso estabelecida e resultados da intervenção. Resultados: Ambas as bases resultaram em 241 artigos, sendo 148 na Pubmed e 93 na Embase. Desse total 82 artigos foram selecionados para leitura na íntegra e após a análise quatro manuscritos foram selecionados, sendo que um manuscrito era duplicado restando três artigos para revisão. Os estudos analisados foram realizados em países desenvolvidos (EUA, Reino Unido e Suíça). Conclusão: Os resultados da revisão sistemática indicam a efetividade desse tipo de programa em estudos de média e longa duração. Também revelam a necessidade de mais estudos científicos devido à escassez de intervenções presenciais com metas de perda de peso em adultos com IMC acima de $30 \mathrm{~kg} / \mathrm{m}^{2}$ e que diferenciem os resultados dos grupos intervenção e controle.
\end{abstract}

Palavras-chave: Tratamento multiprofissional; Desfecho primário; Obesidade.

\begin{abstract}
Introduction: The increase in overweight, obesity and severe obesity in the world represents a major public health challenge in terms of both prevention and treatment of obesity and associated chronic diseases. Objective: This review analyzes multiprofessional studies whose goals include at least 5\% weight loss used in programs aimed at changing behavior in adults with a BMI above $30 \mathrm{~kg} / \mathrm{m} 2$ as well as their clinical outcomes. Methods: A systematic review was carried out in the Pubmed and Embase databases with the keywords "multi-professional", "treatment", "primary care" and "obesity", in both bases the filters "clinical trial", "studies randomized "and" adults over 18 years ", the selected works should have evaluated face-to-face intervention programs, with an established weight loss goal and intervention results. Results: Both databases resulted in 241 articles, 148 in Pubmed and 93 in Embase. Of this total, 82 articles
\end{abstract}


were selected for reading in full and after analysis four manuscripts were selected, with one manuscript being duplicated, leaving three articles for review. The analyzed studies were carried out in developed countries (USA, United Kingdom and Switzerland). Conclusion: The results of the systematic review indicate the effectiveness of this type of program in studies of medium and long duration. They also reveal the need for more scientific studies due to the scarcity of face-to-face interventions with weight loss goals in adults with a BMI above $30 \mathrm{~kg} / \mathrm{m} 2$ and that differentiate the results of the intervention and control groups.

Keywords: Multi-professional treatment; Primary care; Obesity.

\begin{abstract}
Resumen
Introducción: El aumento del sobrepeso, la obesidad y la obesidad severa en el mundo representa un importante desafío de salud pública en términos de prevención y tratamiento de la obesidad y las enfermedades crónicas asociadas. Objetivo: Esta revisión analiza estudios multiprofesionales cuyos objetivos incluyen al menos una pérdida de peso del 5\% utilizada en programas destinados a cambiar el comportamiento en adultos con un IMC superior a 30 $\mathrm{kg} / \mathrm{m} 2$, así como sus resultados clínicos. Métodos: Se realizó una revisión sistemática en las bases de datos Pubmed y Embase con las palabras clave "multiprofesional", "tratamiento", "atención primaria" y "obesidad", en ambas bases

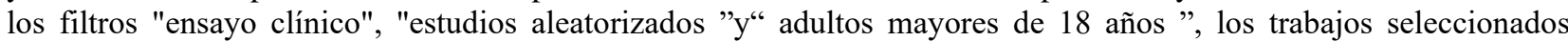
deben haber evaluado programas de intervención presencial, con un objetivo de pérdida de peso establecido y resultados de la intervención. Resultados: Ambas bases de datos dieron como resultado 241 artículos, 148 en Pubmed y 93 en Embase. De este total, se seleccionaron 82 artículos para lectura en su totalidad y luego del análisis se seleccionaron cuatro manuscritos, con un manuscrito duplicado, dejando tres artículos para revisión. Los estudios analizados se realizaron en países desarrollados (EE.UU., Reino Unido y Suiza). Conclusión: Los resultados de la revisión sistemática indican la efectividad de este tipo de programas en estudios de mediana y larga duración. También revelan la necesidad de más estudios científicos por la escasez de intervenciones presenciales con objetivos de adelgazamiento en adultos con IMC superior a $30 \mathrm{~kg} / \mathrm{m} 2$ y que diferencien los resultados de los grupos de intervención y control.
\end{abstract}

Palabras clave: Tratamiento multiprofesional; Resultado primario; Obesidad.

\title{
1. Introdução
}

A complexidade da obesidade está associada a várias doenças crônicas importantes, incluindo doenças cardiovasculares, diabetes tipo 2 e câncer (Lee et al., 2016), além disso pode contribuir para distúrbios psicológicos, incluindo depressão e estigmatização social (Apovian et al., 2015). A prevalência mundial da obesidade segundo a Organização Mundial da Saúde quase triplicou entre 1975 e 2016 (WHO, 2017) e esse aumento resultou em uma alta prevalência tanto em países desenvolvidos como em desenvolvimento (Morgen \& Sørensen, 2014).

$\mathrm{Na}$ América Latina e no Caribe um em cada quatro adultos convivem com obesidade e, todos os anos, 600 mil pessoas morrem nessas regiões em decorrência das doenças relacionadas à má alimentação, diabetes, hipertensão e doenças cardiovasculares (OPAS, 2020). O aumento mais expressivo da obesidade adulta foi observado no Caribe, onde o percentual quadruplicou, passando de 6\% em 1975 para 25\%, um aumento em termos absolutos de 760 mil para 6,6 milhões de pessoas (OPAS, 2020). As projeções da OECD até 2030 indicam um aumento constante nas taxas de obesidade. Se nada for feito os países como Estados Unidos, México e Inglaterra apresentarão em termos absolutos respectivamente 47\%, 39\% e 35\% da população com obesidade (OECD, 2017).

O tratamento não é uma tarefa fácil, a obesidade é uma doença crônica complexa com muitos fatores contribuintes, por isso é importante iniciar o tratamento o quanto antes, pois alterações decorrentes do excesso de peso são cada vez mais frequentes ainda na fase infanto-juvenil (Castilho et al., 2021; Dietz, 2001). Os médicos da atenção primária e especialistas em obesidade podem orientar os tratamentos que incluem abordagens de estilo de vida, como dieta, exercícios físicos e abordar os fatores emocionais que contribuem para a obesidade. No entanto, para algumas pessoas, a cirurgia para perda de peso poderá ser uma necessidade (Tello, 2018).

A literatura tem apontado que a perda de peso passa a ser significativa, com redução de comorbidades, e apresentar benefícios para saúde a partir de 5 a 10\% de seu peso inicial (Garvin, 2015; Jensen et al., 2014; Kolasa et al., 2010). Os principais componentes para alcançar o sucesso clínico incluem prescrição de dieta moderadamente reduzida em calorias, 
progressivo aumento da atividade física, estratégias comportamentais para ajudar na adesão ao programa e intervenções com no mínimo 6 meses, pois os participantes encontrarão um equilíbrio calórico e energético após esse tempo e consequentemente realizarão novos ajustes para a continuidade da perda de peso (Jensen et al., 2014). Essa meta pode ajudar a diminuir os riscos de problemas crônicos de saúde como doenças cardíacas e diabetes tipo 2 (Mayo Clinic, 2016). O que provavelmente leva a diminuição do risco de morte prematura relacionado a condições crônicas graves e que também mudaria a qualidade de vida da população (Christinelli et al., 2021; WHO, 2003).

A comunidade cientifica começou a dar destaque para o desenvolvimento de metas na década de 80 (Strecher et al., 1995), sendo o Bandura e Simon (1977) um dos primeiros estudos encontrado na literatura que trabalhou o componente de educação, manual de autocontrole, definição de metas para reduzir em 10\% a ingestão de alimentos a cada quatro semanas e, com os participantes sendo automonitorados. O estudo era composto por 9 homens e 57 mulheres com idade média de 47 anos que tinham sobrepeso ou obesidade e não tinham controle sobre seu comportamento alimentar (Bandura \& Simon, 1977).

Mais recentemente outros estudos mostraram que o estabelecimento de metas é importante para induzir o participante da pesquisa a mudar seus hábitos e levar uma vida mais saudável como o estudo de Kinsey et al., (2018), que evidenciou bons resultados em mulheres adultas afro-americanas e brancas com sobrepeso ou obesas (Kinsey et al., 2018). Patel et al., (2019) mostraram que a dieta, o uso de metas personalizadas e um aplicativo móvel comercial fizeram com que os participantes tivessem uma perda de peso clinicamente significativa (Patel et al., 2019). Xu et al., (2017) demostraram resultados estatisticamente significativos em relação as metas de perda de peso, adesão e atividade física em uma coorte de 239 participantes com obesidade de ambos os sexos, acompanhados por automonitoramento (Xu et al., 2017).

De acordo com Strecher et al., (1995) a teoria do estabelecimento de metas prevê que, sob certas condições, o estabelecimento de metas difíceis específicas leva a um desempenho superior quando comparado com nenhuma meta ou metas vagas e não quantitativas, como "faça o seu melhor". O autor também descreve três mecanismos motivacionais pelos quais o estabelecimento de metas tem um efeito benéfico no desempenho: o esforço, persistência e concentração e além disso leva a pessoa a se esforçar mais por longos períodos de tempo, com menos distração do objetivo (Strecher et al., 1995).

Neste contexto, Strecher et al., (1995) recomenda que o desenvolvimento de estratégias e definição de metas sejam ofertadas em programas de mudança de comportamento visando melhorias de saúde, por esse motivo, programas de intervenção multiprofissionais voltados para o tratamento de problemas de saúde têm demostrado bons resultados clínicos por abordarem aspectos relacionados à prática regular de exercícios físicos (Strecher et al., 1995), questões nutricionais e psicossociais em pessoas com diferentes níveis de sobrepeso e obesidade tanto para crianças e adolescentes (Bianchini et al., 2012), adultos (Castilho et al., 2020, Castilho et al., 2021; Crowe et al., 2015) e também para idosos (Haywood \& Sumithran, 2019).

Pesquisas sobre o tratamento da obesidade que foquem na meta de perda de peso a partir de $5 \%$ em adultos com obesidade e que se baseiam em um programa de tratamento que os auxilie na aquisição dessas mudanças são de fundamental importância em decorrência dos benefícios para a saúde, e assim prevenir gastos farmacológicos e cirúrgicos. Com isso, o objetivo do estudo é investigar programas de intervenção que monitoraram as atividades, apresentaram o desfecho clínico do programa das intervenções e que tenham proposto metas de perda de peso a partir de $5 \%$ do peso inicial.

\section{Metodologia}

A revisão sistemática foi realizada seguindo as diretrizes do PRISMA que ajuda os autores a melhorarem o relato da revisão e diminuir o risco de viés. O estudo foi cadastrado, com o número de registro CRD42020205982, no PROSPERO que 
consiste em um registro de protocolo de revisões sistemáticas que permite avaliar a condução do estudo e a qualidade do relato dos resultados, aumentando a transparência e a reprodutibilidade desses estudos.

As bases de buscas utilizadas foram: Medline (via PubMed) que integra um sistema Online de Busca e Análise de Literatura Médica e Embase sendo um sistema Online de Busca e Análise de Literatura biomédica e farmacêutica. As seguintes palavras-chave foram usadas para a pesquisa: "multi-professional", "treatment", "primary care" e "obesity". Operadores booleanos e filtros de pesquisa foram utilizados em ambas as bases de dados para melhorar a pesquisa eletrônica como: ensaio clínico randomizado, teste controlado e aleatório, humanos e adultos maiores de 18 anos.

Em decorrência dos limitados estudos encontrados que se encaixaram nos critérios de inclusão, optamos por não limitar o tempo de publicação, uma vez que reduziria ainda mais os resultados encontrados. A revisão iniciou no mês de março de 2020 e terminou em dezembro do mesmo ano. Estudos adicionais de interesse foram identificados sem ajuda de software apenas por pesquisas bibliográficas e que foram apresentadas como estudos semelhantes.

Os critérios de inclusão na revisão exigiam que os estudos utilizassem o estabelecimento de metas na intervenção para o tratamento clínico dos participantes como um desfecho primário da perda de peso ou do IMC, direcionados a adultos com idade maior ou igual 18 anos que tivessem IMC $\geq 30 \mathrm{~kg} / \mathrm{m}^{2}$, a intervenção teria que ter um acompanhamento de profissionais da área da saúde que monitorassem e/ou auxiliassem nos programas de atividades físicas, psicologia, nutrição entre outros. Estudos que apresentassem intervenções com crianças e adolescentes, que se baseassem apenas na restrição calórica, sem atividade física monitorada e programas apoiados em intervenções farmacológicas para perda de peso ou que já tivessem feito cirurgia de redução do estomago foram excluídos.

As buscas foram realizadas por dois pesquisadores (I.A.S.P) e (N.N.J) de forma independente para determinar a elegibilidade do estudo. Ao final as diferenças de opinião entre os pesquisadores sobre a inclusão do estudo foram resolvidas por consenso. A busca bibliográfica resultou em 241 artigos científicos somando ambas as bases, na Pubmed resultou em 148 artigos e após os filtros "ensaio clínico", "estudos randomizados" e "adultos maiores de 18 anos" resultou em 38 artigos e desses todos foram selecionados para leitura na íntegra, na Embase resultou em 93 artigos e após os filtros "ensaio clínico", "estudos randomizados" e "adultos maiores de 18 anos" 44 foram selecionados para leitura. Após a análise apenas 3 manuscritos foram selecionados para a presente revisão. Os demais foram excluídos por abordarem intervenções sem atividade física, sendo que a grande maioria dos estudos que a atividade física não era monitorada, apenas recomenda.

A Figura 1 apresenta o processo de seleção dos artigos científicos para análise da revisão sistemática. 
Figura 1. Fluxograma do número de artigos encontrados e selecionados após aplicação dos critérios de inclusão e exclusão.

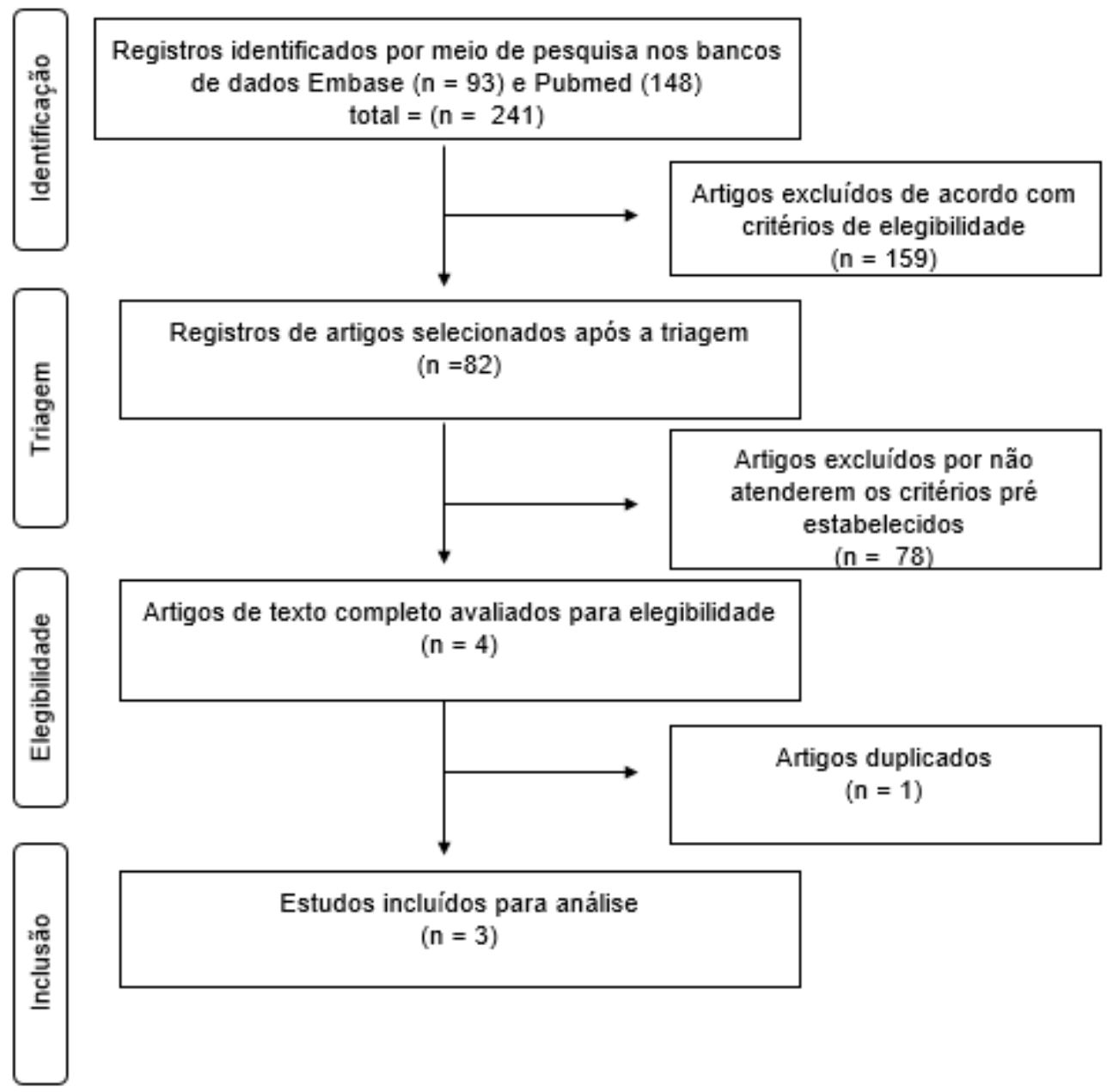

Fonte: Autores.

\section{Resultados}

Os estudos selecionados para a presente revisão foram incluídos com base em vários conjuntos de critérios que os participantes teriam que apresentar no programa de tratamento. Os autores selecionaram três manuscritos que correspondiam aos critérios de inclusão pré estabelecidos para leitura na íntegra e análise completa. O número reduzido de estudos encontrado para revisão demostra a importância de focar em pesquisas com essa população que evidenciam programas multiprofissionais com IMC acima de $30 \mathrm{~kg} / \mathrm{m}^{2}$, que tenha intervenção presencial e metas que estimule o participante a perder peso.

O primeiro trabalho a ser discutido é do Milsom et al., (2011) que foi desenvolvido por pesquisadores dos Estados Unidos (EUA) e o estudo teve intervenção apenas com mulheres que residiam em áreas rurais. A média de IMC foi de 36,8 \pm 5,0 caracterizando obesidade grau II (Milsom et al., 2011). As participantes foram acompanhadas por profissionais de Nutrição, Psicologia e Educação Física durante a intervenção para passar as recomendações do programa, conforme a tabela 1.

As participantes tiveram seis meses de intervenção presencial que contou com 24 sessões e após a conclusão elas foram direcionadas para um dos três grupos de intervenção: face a face (presencial) duas vezes no mês, chamada telefônica de 10 a 15 minutos com duas ligações no mês ou mensagem por correio que nesse caso recebiam os boletins com informações do programa quinzenalmente, essa etapa durou 12 meses de intervenção. As que concluíram essa etapa realizando todas as avaliações eram convidadas a participar da última etapa por meio do acompanhamento (follow-up) que duraria mais 30 meses, mas sem o contato dos profissionais. 
O programa incluiu metas de redução de ingestão energética de 500-1000 Kcal/dia, redução de gorduras totais para 25 a 30\%, redução da proteína para 15\% da ingestão calórica total, metas de atividade física com adição de mais de 3000 passos por dia acima do registro na avaliação inicial (linha de base) ou 30 minutos de caminhada por dia de acordo com a Tabela 2.

Das 234 mulheres incluídas na pesquisa 110 concluíram o acompanhamento inicial de seis meses e apresentaram uma redução média de peso de $10,17 \% \pm 5,0 \%$. A segunda parte da pesquisa que durou 12 meses os autores não diferenciaram os resultados dos três grupos avaliados o que resultou em um ganho médio de $1,73 \pm 6,03 \mathrm{~kg}$ e a última etapa que durou 30 meses sem o contato dos profissionais houve um adicional de 4,10 \pm 7,74 kg. Entretanto, considerando o período total de intervenção de três anos e meio as participantes tiveram uma redução de 4,08\% e 46 mulheres reduziram mais de 5\% como mostra a tabela 3.

Jennings et al., (2014) realizaram a pesquisa no Reino Unido com 230 pessoas de ambos os sexos com base nos serviços desenvolvidos na prática médica. O intuito do programa era facilitar a perda de peso por meio das mudanças progressivas e sustentáveis no estilo de vida, de acordo com as metas individuais elaboradas ao longo de um programa de 1 ano. O programa recrutou participantes maiores de 18 anos com IMC $\geq 40 \mathrm{~kg} / \mathrm{m}^{2}$ ou IMC $\geq 30 \mathrm{~kg} / \mathrm{m}^{2}$ com comorbidade relacionada à obesidade e / ou circunferência da cintura $\geq 102 \mathrm{~cm}$ em homens ou $\geq 88 \mathrm{~cm}$ em mulheres. Os critérios de exclusão eram gravidez, transtorno alimentar grave, falta de motivação ou não responder o contato do programa (Jennings et al., 2014).

A intervenção contou com profissionais de várias áreas como médicos, nutricionistas, psicólogos, educador físico, instrutor de saúde e consultor de saúde. A média de IMC foi de $44,1 \pm 7,8 \mathrm{~kg} / \mathrm{m}^{2}$ o que caracteriza obesidade severa e a média de idade foi de $52 \pm 13,6$ anos conforme Tabela 1 .

A intervenção durou 12 meses para 213 participantes e 17 tiveram intervenção intensiva de seis meses. Após esse período os participantes que continuaram no programa foram acompanhados até completarem dois anos finalizando com 84 participantes. No período de um ano os autores informaram que nenhum participante tinha sido submetido a cirurgia bariátrica e com a conclusão dos dois anos apenas duas pessoas foram submetidas ao procedimento cirúrgico.

As aulas de Educação Física eram ministradas por um profissional de forma individual e em pequenos grupos e também havia um encaminhamento de exercícios de 12 semanas em academias locais. O grupo de intervenção intensiva teve 15 visitas presencias e o grupo de longo prazo recebeu de 10 a 15 visitas presenciais. Os profissionais orientavam e ajustavam o programa que incluía dieta individual estruturada e aulas de educação voltadas ao tratamento do peso.

Os resultados desse estudo mostraram que dos 230 participantes recrutados 117 completaram o período de um ano e aos dois anos 45 participantes compareceram as avaliações da intervenção com uma taxa de evasão de 80,1\% no período de follow-up. O percentual dos 170 participantes os quais concluíram a intervenção de um ano que tiveram 5\% ou mais de perda de peso foi de 25,2\% aos 3 meses, 44,1\% aos 6 meses, 59,1\% aos 9 meses e 60\% dos participantes aos 12 meses. Aos 2 anos a perda de peso média foi de $5 \mathrm{~kg}$ com $44,4 \%$ perdendo $5 \%$ de seu peso. A qualidade de vida avaliada pelo questionário EQ-5D$5 \mathrm{~L}$ indicou melhores resultados, redução da pressão arterial e nos valores de HbA1c em diabéticos também foram constatados ao final do período de um ano como mostra a tabela 3.

Já o estudo de Pjanic et al., (2017) que foi realizado na Suíça e contou com a participação de 238 pessoas (191 mulheres e 47 homens) com idade média de $41 \pm 12,3$ anos e IMC $40,1 \mathrm{~kg} / \mathrm{m}^{2}$ para o tratamento em grupo e $38,9 \mathrm{~kg} / \mathrm{m}^{2}$ para o tratamento individual (Pjanic et al., 2017). O programa em grupo contou com a participação de Nutricionistas, Psicólogos, profissional de consciência corporal e Médicos como indica a Tabela 1.

A intervenção durou 12 meses sendo que os primeiros seis meses foram mais intensos com uma sessão semanal de 90 minutos em terapia cognitivo-comportamental, três sessões de 90 minutos em aconselhamento nutricional, uma sessão semanal de duas horas com profissional de consciência corporal e duas sessões de exercícios físicos variados de 60 minutos. As 
semanas de 7 a 12 os participantes receberam a intervenção de forma alternada de treinamento cognitivo comportamental ou aconselhamento nutricional e uma sessão semanal de esportes variados de 60 minutos (entre as atividades hidroginástica, caminhada nórdica e treinamento com pesos na academia do hospital), Tabela 2.

O programa individual constituiu-se em sessões mensais com nutricionistas e médico, mas de forma voluntária os participantes poderiam frequentar as aulas de consciência corporal em um total de oito sessões semanais e/ou tratamento psicológico em sete sessões semanais junto com o tratamento em grupo. E além disso os participantes que necessitassem poderiam receber tratamento adicional de nutricionista e médico, Tabela 2.

Os resultados indicam que os participantes tiveram uma perda de peso média de $2,5 \mathrm{~kg}$ aos três meses e $4,2 \mathrm{~kg}$ em um ano. O IMC estava em $39,6 \mathrm{~kg} / \mathrm{m}^{2}$ no momento pré, $38,5 \mathrm{~kg} / \mathrm{m}^{2}$ aos três meses e $37,7 \mathrm{~kg} / \mathrm{m}^{2}$ aos 12 meses. Os resultados são apresentados em conjunto, pois não diferiram significativamente entre o tratamento individual e em grupo ( $\mathrm{p}=0,08)$. Dos 233 participantes aos três meses 20,6\% haviam perdidos 5\% do seu peso e após um ano dos 179 participantes 41,4\% perderam no mínimo 5\% do peso corporal e também reduziram a depressão e a vergonha avaliados por questionários como indica a Tabela 3. 
Tabela 1. Artigos analisados e ordenados por ano de publicação.

\begin{tabular}{|c|c|c|c|c|c|}
\hline Autor/Ano & Origem & Áreas envolvidas & $\begin{array}{l}\text { Gênero (Feminino/ } \\
\text { Masculino) }\end{array}$ & $\begin{array}{l}\text { Idade (Média } \pm \text { Desvio } \\
\text { Padrão) }\end{array}$ & IMC Inicial \\
\hline Milsom (2011) & EUA & $\begin{array}{l}\text { 1) Nutrição } \\
\text { 2) Psicologia } \\
\text { 3) Educação Física }\end{array}$ & 234 mulheres & $59,3 \pm 6,2$ & $36,8 \pm 5,0$ \\
\hline Jennings (2014) & Reino Unido & $\begin{array}{l}\text { 1) Médico bariátrico. } \\
\text { 2) Nutricionista. } \\
\text { 3) Psicólogo. } \\
\text { 4) Profissionais de } \\
\text { Educação Física. } \\
\text { 5) Instrutor de saúde. } \\
\text { 6) Médico } \\
\text { endocrinologista. } \\
\text { 7) Consultor de saúde } \\
\text { pública. }\end{array}$ & $\begin{array}{l}\text { Total }=230(161 \\
\text { Mulheres e } 69 \text { Homens })\end{array}$ & $52 \pm 13,6$ & $44,1 \pm 7,8$ \\
\hline Pjanic (2017) & Suíça & $\begin{array}{l}\text { 1) Nutricionistas, } \\
\text { 2) Psicólogos, } \\
\text { 3) Profissional de } \\
\text { Educação Física } \\
\text { 4) Médicos }\end{array}$ & $\begin{array}{l}\text { Total }=238(191 \\
\text { mulheres e } 47 \text { homens })\end{array}$ & $41 \pm 12,3$ & $\begin{array}{l}\text { IMC do Tratamento em } \\
\text { grupo }=40,1 \\
\text { IMC do grupo tratamento } \\
\text { individual }=38,9\end{array}$ \\
\hline
\end{tabular}

Fonte: Autores. 
Tabela 2. Descrições dos estudos de definição de metas a partir de 5\% direcionados a adultos com obesidade.

\begin{tabular}{|c|c|c|c|c|}
\hline Autores e ano & $\begin{array}{c}\text { Duração do programa de } \\
\text { intervenção }\end{array}$ & Objetivo do estudo & Detalhes da intervenção & $\begin{array}{l}\text { Frequência das } \\
\text { avaliações }\end{array}$ \\
\hline Milsom (2011) & $\begin{array}{l}\text { Primeiros } 6 \text { meses ( } 24 \text { sessões) } \\
\text { presenciais. Depois direcionados } \\
\text { para } 3 \text { anos de uma das } \\
\text { intervenções (aconselhamento face } \\
\text { a face, aconselhamento por telefone } \\
\text { ou contato apenas por correio) }\end{array}$ & $\begin{array}{l}\text { Examinar os fatores } \\
\text { comportamentais } \\
\text { associados ao sucesso na } \\
\text { manutenção do peso a } \\
\text { longo prazo entre os } \\
\text { participantes que } \\
\text { completaram uma } \\
\text { intervenção inicial de seis } \\
\text { meses. }\end{array}$ & $\begin{array}{l}\text { - } \quad \text { Cada sessão durou em média } 90 \text { minutos } \\
\text { - Metas de redução na ingestão de energia em } 500-1000 \text { kcal / } \\
\text { - } \quad \text { Metas de redução nas gorduras totais para } 25 \% \text { a } 30 \% \\
\text { - Metas redução da proteína para } 15 \% \text { da ingestão calórica total } \\
\text { - As metas de atividade física incluíram a adição de mais de } \\
\text { 3.000 passos por dia acima da linha de base, ou } 30 \text { minutos / } \\
\text { dia de caminhada, seis dias / semana. } \\
\text { - Desfecho primário de redução de pelo menos } 5 \% \text { do peso. }\end{array}$ & $\begin{array}{l}\text { Quatro avaliações } \\
\text { (pré - } 6 \text { meses - } 18 \\
\text { meses - } 48 \text { meses) }\end{array}$ \\
\hline $\begin{array}{l}\text { Jennings } \\
(2014)\end{array}$ & $\begin{array}{l}213 \text { participantes receberam } \\
\text { intervenção de } 12 \text { meses e } 17 \\
\text { participantes receberam intervenção } \\
\text { de } 6 \text { meses. Após a conclusão } \\
\text { foram acompanhados até } \\
\text { completarem } 2 \text { anos }\end{array}$ & $\begin{array}{l}\text { O desfecho primário foi a } \\
\text { perda de peso }>5 \% \text { em } 1 \\
\text { ano, com o objetivo de } \\
\text { atingir essa meta em } 6 \\
\text { meses em } 50 \% \text { dos } \\
\text { participantes. }\end{array}$ & $\begin{array}{l}\text { - O grupo de financiamento individual }(\mathrm{N}=17) \text { recebeu } \\
\text { Intervenção de } 6 \text { meses com } 15 \text { visitas presencias. } \\
\text { O grupo de longo prazo }(\mathrm{N}=213 \text { e } 12 \text { meses }) \text { recebeu } \\
\text { Intervenção de } 10 \text { a } 15 \text { visitas presenciais. } \\
\text { O programa oferecia sessões individuais e também em grupos } \\
\text { na academia e além disso, havia um esquema de } \\
\text { encaminhamento de exercícios de } 12 \text { semanas em academias } \\
\text { locais. } \\
\text { - Perda de peso alvo } \geq 5 \%\end{array}$ & $\begin{array}{l}\text { Cinco avaliações } \\
\text { (pré - } 3 \text { meses - } 6 \\
\text { meses - } 9 \text { meses - } \\
12 \text { meses - } 18 \\
\text { meses - } 2 \text { anos) }\end{array}$ \\
\hline Pjanic (2017) & 12 meses & $\begin{array}{l}\text { Relatar os efeitos dos } \\
\text { resultados a longo prazo } \\
\text { do programa } \\
\text { interdisciplinar em relação } \\
\text { à mudança de peso, estado } \\
\text { emocional e habilidades } \\
\text { de regulação emocional. }\end{array}$ & $\begin{array}{l}\text { - Durante as semanas } 1 \text { a } 6 \text { : uma sessão de } 90 \text { minutos de } \\
\text { terapia cognitiva comportamental, três sessões de } 90 \text { min em } \\
\text { aconselhamento nutricional, uma sessão semanal de } 2 \mathrm{~h} \text { de } \\
\text { consciência corporal e duas sessões de exercícios físicos de } 60 \\
\text { min. } \\
\text { Durante as semanas de } 7 \text { a } 12 \text { : Sessões semanais alternadas de } \\
\text { terapia cognitivo-comportamental ou aconselhamento } \\
\text { nutricional e } 1 \text { sessão semanal de esportes de } 60 \text { minutos } \\
\text { Abordou a definição de metas realistas de peso } \geq 5 \%\end{array}$ & $\begin{array}{l}\text { Três avaliações } \\
\text { (pré - } 3 \text { meses - } 1 \\
\text { ano) }\end{array}$ \\
\hline
\end{tabular}


Tabela 3. Resultados estatísticos das intervenções dos artigos avaliados.

\begin{tabular}{|c|c|c|c|c|c|c|c|}
\hline Estudo & $\begin{array}{l}\text { Participante } \\
\text { concluintes/ Média } \\
\text { da faixa etária }\end{array}$ & $\begin{array}{l}\text { Resultados } \\
\text { antropométricos }\end{array}$ & $\begin{array}{l}\text { Resultados } \\
\text { bioquímicos }\end{array}$ & $\begin{array}{l}\text { Resultados } \\
\text { psicológicos }\end{array}$ & $\begin{array}{l}\text { Resultados } \\
\text { hemodinâmicos }\end{array}$ & Perda de peso & $\begin{array}{l}\text { (N) Perda } \geq 5 \% \text { do } \\
\text { peso }\end{array}$ \\
\hline Milson (2011) & $\begin{array}{l}N=110 / \\
60,08 \pm 6,17 \text { anos }\end{array}$ & $\left.\begin{array}{l}\text { Peso } \\
\text { IMC }\end{array}\right]$ & $*$ & $*$ & $*$ & $\begin{array}{l}\text { Não houve } \\
\text { diferença } \\
\text { significativa } \\
\text { Entre os grupos. } \\
\text { Nos primeiros } 6 \\
\text { meses perderam no } \\
\text { conjunto } 10,17 \% \pm \\
5,0 \% \text { do peso. } \\
\text { Do mês } 0 \text { ao } 48 \\
\text { perderam } 4,08 \% \text { do } \\
\text { peso. }\end{array}$ & $\begin{array}{l}41,80 \%(\mathrm{n}= \\
46) \text { atingiram a } \\
\text { meta de perda de } \\
\text { peso em } 3,5 \text { anos. }\end{array}$ \\
\hline Jennings (2014) & $\begin{array}{l}\mathrm{N}=84 / \\
57,5 \text { anos }\end{array}$ & $\left.\begin{array}{l}\text { Peso } \\
\mathrm{IMC} \\
\mathrm{CC}\end{array}\right]$ & HbA1c $]$ & Qualidade de vida $\uparrow$ & Pressão arterial & $\begin{array}{l}\text { Do mês } 0 \text { aos } 18 \\
\text { meses houve uma } \\
\text { redução de } 7,1 \% \\
(\mathrm{DP} 9,5)(\mathrm{N}=157) \\
\text { do peso e aos } 2 \text { anos } \\
\text { um média de }-5 \mathrm{Kg} \\
(\mathrm{N}=84)\end{array}$ & $\begin{array}{l}34,2 \%(\mathrm{~N}=117) \text { com } \\
\text { três meses; } 53,8 \% \\
(\mathrm{~N}=117) \text { com seis } \\
\text { meses; } \\
65,8 \%(\mathrm{~N}=117) \text { com } \\
\text { nove meses; } 72,6 \% \\
(\mathrm{~N}=117) \text { com doze } \\
\text { meses. }\end{array}$ \\
\hline Pjanic (2017) & $\begin{array}{l}\mathrm{N}=179 / \\
41,4 \text { anos }\end{array}$ & $\left.\begin{array}{l}\text { Peso } \\
\text { IMC }\end{array}\right]$ & $*$ & $\begin{array}{l}\text { Depressão e } \\
\text { vergonha }\end{array}$ & $*$ & $\begin{array}{l}\text { Do mês } 0 \text { a } 3 \\
\text { perderam } 2,5 \mathrm{~kg} \\
(\mathrm{IQR} 0,7-5,1 \mathrm{~kg}) \\
(\mathrm{N}=233) . \text { Após } 1 \\
\text { ano a perda de peso } \\
\text { foi de } 4,2 \mathrm{~kg} \text { (IQR } \\
1,1-8,6 \mathrm{~kg}) \\
(\mathrm{N}=179) .\end{array}$ & $\begin{array}{l}\text { Após } 3 \text { meses } \\
20,6 \%(\mathrm{~N}=233) \\
\text { haviam perdido } \geq \\
5 \% \text { do peso. Após } 1 \\
\text { ano } 41,4 \%(\mathrm{~N}= \\
179) \text { perderam } \geq \\
5 \% \text { do peso. }\end{array}$ \\
\hline
\end{tabular}




\section{Discussão}

Com os crescentes aumentos de prevalência da obesidade no mundo, intervenções multiprofissionais com foco na redução de peso por meio de técnicas comportamentais são fundamentais. O objetivo da presente revisão foi analisar artigos com o estabelecimento de metas que tivessem intervenção presenciais com diferentes profissionais da área da saúde visando a redução do peso corporal em, no mínimo $5 \%$ do peso inicial dos participantes.

Todos os estudos avaliados apresentaram estratégias práticas de intervenção visando o desfecho clínico perda de peso. O tempo de intervenção não foi a variável mais importante para reduzir 5\% ou mais do peso corporal junto a essa população de adultos com obesidade, mas sim a estratégia utilizada, assim como mostra Johnston et al (2018) o que diferenciou os participantes que atingiram uma redução de $\geq 10 \%$ do peso inicial em seis meses em três grupos diferentes, sendo que $65,5 \%$ dos participantes que compareceram em reuniões presencias atingiram essa meta, o uso de site para automonitoramento $41,6 \%$ dos participantes e uso de aplicativo móvel apenas 17,7\% dos participantes conseguiram perder $\geq 10 \%$ do seu peso inicial (Johnston et al., 2019) . Essa redução é importante para diminuir as comorbidades que enfrentam esse público adulto e além disso é um fator importante que indica que o tempo de intervenção não é o mais influente para trazer benefícios à saúde (Garvin, 2015; Jensen et al., 2014; Kolasa et al., 2010).

Em relação ao estudo de Milsom et al (2011) que teve seis meses de intervenção intensiva e após esse período teve um acompanhamento de três grupos que adotaram diferentes estratégias para perda de peso como redução de energia de 500 $1000 \mathrm{kcal} /$ dia, redução de gorduras totais de 25 a 30\%, redução da proteína para 15\% da ingestão calórica total, metas de atividade física fora do ambiente de intervenção com a adição de 3000 passos por dia ou 30 minutos de caminhada por dia (Milsom et al., 2011). Entretanto, o autor não diferenciou a perda de peso por grupo, apenas relatou o resultado final de todos sendo que nos primeiros seis meses foram mais satisfatórios com uma redução média de $10,17 \% \pm 5,0 \%$ e recuperaram $1,73 \pm$ $6,03 \mathrm{~kg}$ do mês 6 ao mês 18 e 4,10 $\pm 7,74 \mathrm{~kg}$ do mês 18 ao mês 48 .

Uma das causas desse ganho de peso pode ser explicada pela mudança da estratégia após os seis meses que passou da intervenção presencial para o acompanhamento on-line, boletins e, para alguns participantes, o contato presencial até 18 meses e dos 18 meses aos 48 não foi ofertado o acompanhamento. Vários artigos mostram que a interação contínua com profissionais da área da saúde ou em ambientes com o contato em grupo proporcionam maior perda de peso e resultados mais expressivos a longo prazo em comparação a intervenções que interrompem o tratamento após um curto período de tempo (Castilho et al., 2021; Hall \& Kahan, 2018).

Já no estudo de Jennings et al (2014) o programa teve duração de 12 meses com acompanhamento de profissionais e mais um ano de follow-up. Os participantes com IMC $\geq 40 \mathrm{~kg} / \mathrm{m}^{2}$ obtiveram bons resultados na qualidade de vida, redução na pressão arterial e nos valores de hemoglobina glicada (HbA1c) entre os diabéticos. Em relação à média do peso perdido em três meses foi de $-4,3 \mathrm{~kg}(\mathrm{n}=152)$, em seis meses $-6,9 \mathrm{~kg}(\mathrm{n}=134)$, nove meses $-9 \mathrm{~kg}(\mathrm{n}=102)$ e 12 meses $-9,3(\mathrm{n}=116)$. Aos dois anos de intervenção 45 participantes concluíram e a perda de peso média foi de - 5kg e 44,4\% mantiveram uma perda de peso acima da meta de 5\% (Jennings et al., 2014).

Resultados satisfatórios em intervenções presenciais com população com obesidade severa também foram observados nos estudo de Crowe et al., (2015); Bim., (2019); Sartorio et al., (2005); Marcon et al., (2011) e Castilho et al., (2021) os quais demostraram após a intervenção presencial, resultados significativos em parâmetros bioquímicos, aptidão física, parâmetros psicológicos, hemodinâmicos, composição corporal, condição cardiometabólica, redução do peso corporal e medidas antropométricas após o período de tratamento intensivo com os profissionais da área da saúde (Bim, 2019; Castilho et al., 
Research, Society and Development, v. 10, n. 1, e24710111779, 2021

(CC BY 4.0) | ISSN 2525-3409 | DOI: http://dx.doi.org/10.33448/rsd-v10i1.11779

2021; Marcon et al., 2011; Sartorio et al., 2005) . Entretanto, esses estudos não entraram na revisão devido ao fato de que não terem estabelecidos metas de perda de peso ao longo do programa.

Em relação ao estudo de Pjanic et al., (2017) a intervenção durou 12 meses, com as primeiras seis semanas sendo mais intensiva. Nesse período, eles tiveram acompanhamento em grupo de terapia cognitivo-comportamental, aconselhamento nutricional, aulas de consciência corporal e exercícios físicos. Após os seis meses alternaram as aulas de terapia cognitivocomportamental ou aconselhamento nutricional com a adição de aulas de esportes. E além disso o autor afirma que houve outro grupo que seria o treinamento individual com aulas mensais com médicos, nutricionistas e também poderiam frequentar aulas de consciência corporal e aulas com psicólogos (Pjanic et al., 2017). No entanto os resultados são apresentados em três momentos sem a diferenciação dos grupos o que dificulta na compreensão de qual foi a melhor estratégia adotada no decorrer da intervenção.

Nos primeiros três meses a média da perda de peso estava entre - 2,5kg e no final aos 12 meses uma perda média de 4,2kg. Resultando dessa forma que dos 233 participantes 20,6\% perderam no mínimo 5\% do seu peso e no final dos 179 participantes que concluíram o estudo, $41,4 \%$ perderam $5 \%$ ou mais de seu peso inicial. Os questionários preenchidos no pós intervenção indicaram redução da depressão e a vergonha que sentiam em relação aos seus corpos (Pjanic et al., 2017).

Isso indica que programas multiprofissionais de tratamento da obesidade (PMTO) são uma opção viável para aqueles que buscam tratamento para melhorar a qualidade de vida e alcançar melhorares resultados clínicos. Por isso esses programas apresentam bons resultados em várias populações seja crianças, adolescentes, adultos ou idosos como já foi discutido anteriormente (Bianchini et al., 2016; Bianchini et al., 2013; Castilho et al., 2021; Lopera et al., 2016; Nardo Junior et al., 2018; Westphal et al., 2020).

Os resultados dos três estudos mostram que a aderência é um problema para os PMTO, pois no artigo de Milsom et al., (2011) o programa iniciou com 110 pessoas e finalizou os 3 anos com 46 participantes, ou seja, uma redução de quase $60 \%$ (Milsom et al., 2011). No estudo de Jennings et al., (2014) o programa iniciou com 230 participantes e finalizou com 84 após dois anos, sendo que a redução foi significativa após os 12 meses que passou de presencial para follow-up com uma redução de 36,5\% (Jennings et al., 2014). Já no estudo de Pjanic et al., (2017) o programa iniciou com 233 participantes e terminou os 12 meses com 179 com uma desistência de 23,1\% (Pjanic et al., 2017). De acordo com alguns estudos a desistência é comum em estudos de longo prazo assim como demostraram os autores Lantz et al., (2003) e Melin et al., (2006) que tiveram acompanhamento de 3 e 4 anos com uma desistência de 51,8 e 53,0\% respectivamente (Lantz et al., 2003; Melin et al., 2006).

Outros indícios sugerem que a desistência pode ser causada devido ao sofrimento psicológico que os participantes têm nas primeiras semanas de tratamento incluindo depressão, ansiedade e baixa autoestima e a falha de atingir as metas propostas nos programas também pode estimular a desistência (Bradshaw et al., 2010; Castilho et al., 2021; Chang et al., 2009; Elfhag \& Rössner, 2010; Fabricatore et al., 2009; Greenberg et al., 2009; Lim et al., 2009; Westphal et al., 2020) . Por esse motivo Colombo et al., (2014) destacam a importância de se valorizar o bem-estar psicológico com cautela a metas de perda de peso, para que pacientes com obesidade continuem participando dos programas de tratamento (Colombo et al., 2014).

Os estudos avaliados em geral apresentaram resultados importantes no combate a obesidade e obesidade severa, embora tenha havido desistências no decorrer do programa, os participantes concluintes apresentaram resultados clínicos satisfatórios. Por esse motivo, faz-se necessário mais estudos com esta população ofertados por PMTO e que tenham a meta de perda de peso, em decorrência do reduzido número de pesquisas encontrados na literatura com esses critérios de inclusão.

Apesar dos resultados satisfatórios apresentados nos estudos, os autores apresentaram algumas limitações da intervenção ao longo do programa. Milsom et al., (2011) destacam a baixa adesão do programa de intervenção de longo prazo 
(3 anos) (Milsom et al., 2011). Pjanic et al., (2017) relataram que as limitações foram a falta de um grupo controle, a alta taxa de abandono da intervenção, o fato de ter incluído apenas participantes que falavam alemão, o estudo havia mais mulheres que homens o que pode ter um risco de viés de gênero e não foi medido a persistência da mudança após um ano de tratamento (Pjanic et al., 2017). Jennigs et al., (2014) destacaram que a principal limitação do programa foi o alto nível de evasão ao fim do programa follow-up o que se tornou um número pequeno dos 18 meses aos dois anos de intervenção com a população de obesidade severa e que um grupo de apoio de longo prazo seria necessário para o próximo programa (Jennings et al., 2014).

Dessa forma, podemos afirmar, com base nos resultados desta revisão, que os programas multiprofissionais com metas de perda de peso têm se mostrado eficazes no tratamento da obesidade. Programas envolvendo a presença de vários profissionais de saúde de diferentes áreas, como Educação Física, Nutrição, Psicologia e Medicina são evidenciados como uma importante abordagem clinica não medicamentosa para o tratamento e prevenção da obesidade e além disso, proporcionam benefícios substancias aos pacientes reduzindo as comorbidades, melhorando a qualidade de vida e reduzindo o risco de mortalidade por todas as causas (Castilho et al., 2020, 2021; Christinelli et al., 2021; Mendes et al., 2016; Westphal et al., 2020).

Com isso sugere-se que novos programas multiprofissionais com metas de perda de peso clinicamente significativas (5\% ou mais) amplie as análises para novos estudos com participantes com obesidade ou obesidade severa, que tenha cuidados com a aderência ao programa e formas de acompanhamento para que se possa avaliar os resultados em longo prazo.

\section{Conclusão}

Existe um número reduzido de estudos na literatura que focou em intervenções presencias, multiprofissionais e com metas de perda de peso a definidas em adultos com IMC $\geq 30 \mathrm{~kg} / \mathrm{m}^{2}$, o que comprova a necessidade de mais investigações sobre essa temática.

Há necessidade de uma estrutura metodológica mais rigorosa sobre os parâmetros de separação dos resultados dos diferentes grupos alocados para intervenção, estudos de mais longa duração que contemplem intervenção presencial com os profissionais da área da saúde, grupo controle e uma amostra mais representativa. Além disso, há necessidade de mais clareza sobre os parâmetros de atividade física como os exercícios realizados, a intensidade do exercício, a frequência e duração e a aptidão física desses participantes.

A presente revisão sistemática mostrou que os programas multiprofissionais com metas de perda de peso foram mais eficientes nas primeiras semanas durante o acompanhamento presencial no combate à obesidade e obesidade severa e suas comorbidades e a desistência foi menor durante esse período.

Ademais, as evidencias sugerem que os programas multiprofissionais de tratamento da obesidade confirmam o sucesso da intervenção e com isso deve-se expandir mais pesquisas sobre essa população para superar possíveis vieses dos estudos analisados nesta revisão sistemática.

\section{Referências}

Apovian, C. M., Garvey, W. T., \& Ryan, D. H. (2015). Challenging obesity: Patient, provider, and expert perspectives on the roles of available and emerging nonsurgical therapies. Obesity, 23(S2), S1-S26. https://doi.org/10.1002/oby.21140

Bandura, A., \& Simon, K. M. (1977). The role of proximal intentions in self-regulation of refractory behavior. Cognitive Therapy and Research, 1(3), 177193. https://doi.org/10.1007/BF01186792

Bianchini, J. A. A., Fernandes Da Silva, D., Lopera, C. A., Rui Matsuo, A., Drieli Seron Antonini, V., \& Nardo, N. (2013). Efectos de un programa multiprofesional de tratamiento de la obesidad sobre los factores de riesgo para síndrome metabólico en niños prepúberes, púberes y adolescentes: Diferencias entre géneros. Revista Andaluza de Medicina del Deporte, 6(4), 139-145. https://doi.org/10.1016/S1888-7546(13)70049-2 
Research, Society and Development, v. 10, n. 1, e24710111779, 2021 (CC BY 4.0) | ISSN 2525-3409 | DOI: http://dx.doi.org/10.33448/rsd-v10i1.11779

Bianchini, J. A. A., Silva, D. F. Da, Lopera, C. A., Antonini, V. D. S., \& Nardo Junior, N. (2016). Intervenção multiprofissional melhora a aptidão física relacionada à saúde de adolescentes com maior efeito sobre as meninas em comparação aos meninos. Revista Brasileira de Educação Física e Esporte, 30(4), 1051-1059. https://doi.org/10.1590/1807-55092016000401051

Bianchini, J., Hintze, L., Bevilaqua, C., Agnolo, C., \& Junior, N. (2012). Tratamento da Obesidade: Revisão de artigos sobre intervenções multiprofissionais no contexto brasileiro. Arq Ciênc Saúde, 19(2), 9-15.

Bim, R. H. (2019). Risco cardiometabólico e tratamento multiprofissional da obesidade em adultos. (Programa de Pós-Graduação em Educação Física UEM/UEL - UEM (org.); Tese).

Bradshaw, A. J., Horwath, C. C., Katzer, L., \& Gray, A. (2010). Non-dieting group interventions for overweight and obese women: What predicts noncompletion and does completion improve outcomes? Public Health Nutrition, 13(10), 1622-1628. https://doi.org/10.1017/S1368980009992977

Castilho, M. M., Westphal, G., Pereira, I. A. S., Martins, F. M., Bim, R. H., Thon, R. A., Moreira, V. F. R., Bevilaqua, C. A., Meireles, K. B., Okawa, R. T. P., \& Júnior, N. N. (2020). Teste de caminhada de 6 minutos (tc6m) na obesidade severa: considerações. Revista Valore, 5(e-555).

Castilho, M. M., Westphal, G., Thon, R. A., Pereira, I. A. S., Martins, F. M., Amaral, M. F. do, Okawa, R. T. P., \& Nardo Junior, N. (2021). Efeitos de um programa multiprofissional de tratamento da obesidade no ambiente aquático em adultos com obesidade severa. Research, Society and Development, 10(1), e12910111636. https://doi.org/10.33448/rsd-v10i1.11636

Chang, M. W., Brown, R., \& Nitzke, S. (2009). Participant recruitment and retention in a pilot program to prevent weight gain in low-income overweight and obese mothers. BMC Public Health, 9. https://doi.org/10.1186/1471-2458-9-424

Christinelli, H. C. B., Westphal, G., Nardo Junior, N., Borim, M. L. C., Costa, M. A. R., \& Fernandes, C. A. M. (2021). Nutritional status and body composition in individuals with overweight or obesity using usual and unusual indicators. Research, Society and Development, 10(1), e4910111339. https://doi.org/10.33448/rsd-v10i1.11339

Colombo, O., Ferretti, V. V., Ferraris, C., Trentani, C., Vinai, P., Villani, S., \& Tagliabue, A. (2014). Is drop-out from obesity treatment a predictable and preventable event? Nutrition Journal, 13(1). https://doi.org/10.1186/1475-2891-13-13

Crowe, C., Gibson, I., Cunningham, K., Kerins, C., Costello, C., Windle, J., O’Shea, P. M., Hynes, M., McGuire, B., Kilkelly, K., Griffin, H., O’Brien, T., Jones, J., \& Finucane, F. M. (2015). Effects of an eight-week supervised, structured lifestyle modification programme on anthropometric, metabolic and cardiovascular risk factors in severely obese adults. BMC Endocrine Disorders, 15(1). https://doi.org/10.1186/s12902-015-0038-x

Dietz, W. H. (2001). The obesity epidemic in young children: Reduce television viewing and promote playing. British Medical Journal, 322(7282), 313-314. https://doi.org/10.1136/bmj.322.7282.313

Elfhag, K., \& Rössner, S. (2010). Initial weight loss is the best predictor for success in obesity treatment and sociodemographic liabilities increase risk for drop-out. Patient Education and Counseling, 79(3), 361-366. https://doi.org/10.1016/j.pec.2010.02.006

Fabricatore, A. N., Wadden, T. A., Moore, R. H., Butryn, M. L., Heymsfield, S. B., \& Nguyen, A. M. (2009). Predictors of attrition and weight loss success: Results from a randomized controlled trial. Behaviour Research and Therapy, 47(8), 685-691. https://doi.org/10.1016/j.brat.2009.05.004

Garvin, J. T. (2015). Weight reduction goal achievement with high-intensity MOVE!@ treatment. Public Health Nursing, 32(3), 232-236. https://doi.org/10.1111/phn.12194

Greenberg, I., Stampfer, M. J., Schwarzfuchs, D., \& Shai, I. (2009). Adherence and success in long-term weight loss diets: The dietary intervention randomized controlled trial (direct). Journal of the American College of Nutrition, 28(2), 159-168. https://doi.org/10.1080/07315724.2009.10719767

Hall, K. D., \& Kahan, S. (2018). Maintenance of Lost Weight and Long-Term Management of Obesity. Medical Clinics of North America, 102(1), 183-197. https://doi.org/10.1016/j.mcna.2017.08.012

Haywood, C., \& Sumithran, P. (2019). Treatment of obesity in older persons-A systematic review. Obesity Reviews, 20(4), 588-598. https://doi.org/10.1111/obr.12815

Jennings, A., Hughes, C. A., Kumaravel, B., Bachmann, M. O., Steel, N., Capehorn, M., \& Cheema, K. (2014). Evaluation of a multidisciplinary Tier 3 weight management service for adults with morbid obesity, or obesity and comorbidities, based in primary care. Clinical Obesity, 4(5), 254-266. https://doi.org/10.1111/cob.12066

Jensen, M. D., Ryan, D. H., Apovian, C. M., Ard, J. D., Comuzzie, A. G., Donato, K. A., Hu, F. B., Hubbard, V. S., Jakicic, J. M., Kushner, R. F., Loria, C. M., Millen, B. E., Nonas, C. A., Pi-Sunyer, F. X., Stevens, J., Stevens, V. J., Wadden, T. A., Wolfe, B. M., \& Yanovski, S. Z. (2014). 2013 AHA/ACC/TOS guideline for the management of overweight and obesity in adults: A report of the American college of cardiology/American heart association task force on practice guidelines and the obesity society. Journal of the American College of Cardiology, 63(25 PART B), 2985-3023. https://doi.org/10.1016/j.jacc.2013.11.004

Johnston, C. A., Moreno, J. P., Hernandez, D. C., Link, B. A., Chen, T. A., Wojtanowski, A. C., Foster, G. D., \& Foreyt, J. P. (2019). Levels of adherence needed to achieve significant weight loss. International Journal of Obesity, 43(1), 125-131. https://doi.org/10.1038/s41366-018-0226-7

Kinsey, A. W., Gowey, M. A., Tan, F., Zhou, D., Ard, J., Affuso, O., \& Dutton, G. R. (2018). Similar weight loss and maintenance in African American and White women in the Improving Weight Loss (ImWeL) trial. Ethnicity and Health, 1-13. https://doi.org/10.1080/13557858.2018.1493435

Kolasa, K. M., Collier, D. N., \& Cable, K. (2010). Weight loss strategies that really work. Journal of Family Practice, 59(7), 378-385. 
Research, Society and Development, v. 10, n. 1, e24710111779, 2021

(CC BY 4.0) | ISSN 2525-3409 | DOI: http://dx.doi.org/10.33448/rsd-v10i1.11779

Lantz, H., Peltonen, M., Agren, L., \& Torgerson, J. S. (2003). A dietary and behavioural programme for the treatment of obesity. A 4-year clinical trial and a long-term posttreatment follow-up. Journal of Internal Medicine, 254(3), 272-279. https://doi.org/10.1046/j.1365-2796.2003.01187.x

Lee, Y., Biddle, S., Chan, M., Cheng, A., Cheong, M., Chong, Y., Foo, L., Lee, C., Lim, S., Ong, W., Pang, J., Pasupathy, S., Sloan, R., Seow, M., Soon, G., Tan, B., Tan, T., Teo, S., Tham, K., \& Wang, J. (2016). Health Promotion Board-Ministry of Health Clinical Practice Guidelines: Obesity. Singapore Medical Journal, 57(8), 472-472. https://doi.org/10.11622/smedj.2016141

Lim, S. S., Norman, R. J., Clifton, P. M., \& Noakes, M. (2009). Psychological Effects of Prescriptive vs General Lifestyle Advice for Weight Loss in Young Women. Journal of the American Dietetic Association, 109(11), 1917-1921. https://doi.org/10.1016/j.jada.2009.08.008

Lopera, C. A., da Silva, D. F., Bianchini, J. A. A., Locateli, J. C., Moreira, A. C. T., Dada, R. P., Thivel, D., \& Nardo, N. (2016). Effect of water- versus landbased exercise training as a component of a multidisciplinary intervention program for overweight and obese adolescents. Physiology and Behavior, 165, 365373. https://doi.org/10.1016/j.physbeh.2016.08.019

Marcon, E. R., Gus, I., \& Neumann, C. R. (2011). Impacto de um programa mínimo de exercícios físicos supervisionados no risco cardiometabólico de pacientes com obesidade mórbida. Arquivos Brasileiros de Endocrinologia e Metabologia, 55(5), 331-338. https://doi.org/10.1590/S000427302011000500006

Mayo Clinic. (2016). Weight loss: 6 strategies for success - Mayo Clinic. Mayo Clinic. https://www.mayoclinic.org/healthy-lifestyle/weight-loss/indepth/weight-loss/art-20047752

Melin, I., Reynisdottir, S., Berglund, L., Zamfir, M., \& Karlström, B. (2006). Conservative treatment of obesity in an academic obesity unit. Long-term outcome and drop-out. Eating and Weight Disorders, 11(1), 22-30. https://doi.org/10.1007/BF03327740

Mendes, A. A., Ieker, A. S. D., de Castro, T. F., Avelar, A., \& Nardo Júnior, N. (2016). Multidisciplinary programs for obesity treatment in Brazil: A systematic review. Revista de Nutricao, 29(6), 867-884. https://doi.org/10.1590/1678-98652016000600011

Milsom, V. A., Middleton, K. M. R., \& Perri, M. G. (2011). Successful long-term weight loss maintenance in a rural population. Clinical interventions in aging, 6, 303-309. https://doi.org/10.2147/cia.s25389

Morgen, C. S., \& Sørensen, T. I. A. (2014). Obesity: Global trends in the prevalence of overweight and obesity. Nature Reviews Endocrinology, 10(9), 513514. https://doi.org/10.1038/nrendo.2014.124

Nardo Junior, N., Bianchini, J. A. A., da Silva, D. F., Ferraro, Z. M., Lopera, C. A., \& Antonini, V. D. S. (2018). Building a response criterion for pediatric multidisciplinary obesity intervention success based on combined benefits. European Journal of Pediatrics, 177(6). https://doi.org/10.1007/s00431-018-31150

OECD, O. for E. C. and D. (2017). Obesity Update. OECD.

OPAS, O. P.-A. D. S. (2020). Nações Unidas pedem ações urgentes para frear o aumento da fome e da obesidade na América Latina e no Caribe. https://www.paho.org/bra/index.php?option=com_content\&view=article\&id=6058

Patel, M. L., Hopkins, C. M., Brooks, T. L., \& Bennett, G. G. (2019). Comparing self-monitoring strategies for weight loss in a smartphone app: Randomized controlled trial. Journal of Medical Internet Research, 21(2). https://doi.org/10.2196/12209

Pjanic, I., Müller, R., Laimer, M., Hagenbuch, N., Laederach, K., \& Stanga, Z. (2017). Evaluation of a multiprofessional, nonsurgical obesity treatment program: which parameters indicated life style changes and weight loss? Journal of Eating Disorders, 5(1), 14. https://doi.org/10.1186/s40337-017-0144-4

Sartorio, A., Maffiuletti, N. A., Agosti, F., \& Lafortuna, C. L. (2005). Gender-related changes in body composition, muscle strength and power output after a short-term multidisciplinary weight loss intervention in morbid obesity. Journal of Endocrinological Investigation, $28(8)$, 494-501. https://doi.org/10.1007/bf03347236

Strecher, V. J., Seijts, G. H., Kok, G. J., Latham, G. P., Glasgow, R., Devellis, B., Meertens, R. M., \& Bulger, D. W. (1995). Goal Setting as a Strategy for Health Behavior Change. Health Education \& Behavior, 22(2), 190-200. https://doi.org/10.1177/109019819502200207

Tello, M. (2018). Obesity is complicated — and so is treating it. Harvard Health Publishing.

Westphal, G., Baruki, S. B. S., Mori, T. A. de, Montebello, M. I. de L., \& Pazzianotto-Forti, E. M. (2020). Effects of Individualized Functional Training on the Physical Fitness of Women with Obesity. Lecturas: Educación Física y Deportes, 25(268), 61-75. https://doi.org/10.46642/efd.v25i268.2084

WHO. (2017). 10 Facts on Obesity. In World Health Organization. https://www.who.int/features/factfiles/obesity/en/

WHO, W. H. O. (2003). World Health Organization What Is Happening? the Global Strategy on Diet, Physical Activity and Health. Cvd, 2-3. https://www.who.int/dietphysicalactivity/media/en/gsfs_general.pdf

Xu, X., Leahey, T. M., Boguszewski, K., Krupel, K., Mailloux, K. A., \& Wing, R. R. (2017). Self-Expansion is Associated with Better Adherence and Obesity Treatment Outcomes in Adults. Annals of Behavioral Medicine, 51(1), 13-17. https://doi.org/10.1007/s12160-016-9823-7 